\title{
HIGIENIZAÇÃO DAS MÃOS NA PREVENÇÃO DE INFECÇÕES CRUZADAS EM UNIDADES DE TERAPIA INTENSIVA
}

HAND HYGIENIZATION TO PREVENT CROSS INFECTIONS IN INTENSIVE CARE UNITS

SILVA, Mariane Costa ${ }^{1}$, SERPA, Priscila Gomes Silva ${ }^{1}$, FURTADO, Renata Lima Lages $^{1 *}$, FURTADO FILHO, Roque Lages ${ }^{1}$. PEREIRA, Ana Karla Galeno1, BELTRÃO, Renata Paula Lima ${ }^{1}$

${ }^{1}$ Faculdade de Ciências Humanas, Exatas e da Saúde do Piauí/Instituto de Educação Superior do Vale do Parnaíba - FAHESP/IESVAP.

*renatafurtado2013.1@gmail.com

\section{RESUMO}

Os pacientes internados em unidade de terapia intensiva (UTI) são constantemente submetidos a procedimentos invasivos, sendo sujeitos a maiores riscos de desenvolver infecções. Os profissionais que atuam nesses setores podem contribuir para a ocorrência de infecções cruzadas, especialmente quando o hábito da higienização das mãos (HM) é feito de modo inadequado ou em quantidade insuficiente. Trata-se de um artigo de revisão de literatura que utilizou como base de dados Scielo, PUBmed, LILACS e BIREME, de onde foram selecionados 107 artigos, depois de aplicar os critérios de inclusão e exclusão, sobraram um total de 15 artigos para base teórica deste trabalho. A HM é uma prática segura e barata no controle das infecções cruzadas, sendo realizada com água e sabão ou com álcool em gel ambos eficazes. A maioria dos artigos selecionados traziam dados sobre enfermeiros e técnicos de enfermagem, demonstrando que estes são profissionais fonte de preocupação e análises constantes no tangente ao assunto; enquanto os fisioterapeutas são reconhecidos pelos bons hábitos quanto prática. Apesar de reconhecer a importância da $\mathrm{HM}$, a adesão ao processo é variável, sendo maior no após o contato com os pacientes, principalmente após algum procedimento, com o risco de contaminação por fluidos. Muitos profissionais não sabem a técnica correta da HM, o que pode contribuir para os altos índices de infecção nas UTI, sendo necessário que os hospitais proporcionem momentos de treinamento e conscientização.

Palavras-chave: Desinfecção das mãos. Unidade de Terapia Intensiva. Infecção. 
SILVA, Mariane Costa; SERPA, Priscila Gomes Silva; FURTADO, Renata Lima Lages; FURTADO FILHO, Roque Lages; PEREIRA, Ana Karla Galeno; BELTRÃO, Renata Paula Lima.

1 ABSTRACT

2 Patients admitted to the intensive care unit (ICU) are constantly submitted to invasive procedures, being subject to greater risks of developing infections. Professionals working in these sectors can contribute to the occurrence of cross-infections, especially when the hand hygiene $(\mathrm{HH})$ habit is done improperly or in insufficient quantities. This is a literature review article that used Scielo, PUBmed, LILACS and BIREME as database, from which 107 articles were selected, after applying the inclusion and exclusion criteria, a total of 15 articles were left for theoretical basis of this work. HH is a safe and inexpensive practice in the control of cross infections, being carried out with soap and water or with alcohol gel, both effective. Most of the selected articles brought data on nurses and nursing technicians, demonstrating that these are professionals of concern and constant analysis regarding the subject, while physiotherapists are recognized for their good habits and practice. Despite recognizing the importance of $\mathrm{MH}$, adherence to the process is variable, being greater after contact with patients, especially after some procedure, with the risk of contamination by fluids. Many professionals do not know the correct HM technique, which can contribute to the high rates of infection in the ICU, requiring hospitals to provide training and awareness moments.

Keywords: Hand disinfection. Infection. Intensive Care Units.

\section{INTRODUÇÃO}

A UTI é o setor hospitalar responsável por cuidar de pacientes em grave estado geral, que necessitam de constante monitorização e da realização frequente de procedimentos invasivos. Devido essa abordagem, os pacientes estão mais propícios ao desenvolvimento de Infecções relacionadas à Assistência à Saúde (IRAS), definidas como infecções que acometem o indivíduo durante a internação ou durante algum procedimento realizado pelas equipes de saúde ${ }^{1,2}$.

Souza et al. (2015) estimam que $15 \%$ das pessoas internadas desenvolvem alguma IRAS, o que resulta no agravamento do quadro e no aumento dos custos com serviços de saúde. Nogueira et al. (2015) alerta que as IRAS adquiridas em ambiente de UTI constituem $20 \%$ de todas as infecções na terapia intensiva, e estimam que $30 \%$ dos pacientes que ficam internados em UTI apresentam pelo menos um processo infeccioso, mais comumente em trato urinário, corrente sanguínea e pulmonar, especialmente as pneumonias associadas à ventilação mecânica ${ }^{3,4}$. 
SILVA, Mariane Costa; SERPA, Priscila Gomes Silva; FURTADO, Renata Lima Lages; FURTADO FILHO, Roque Lages; PEREIRA, Ana Karla Galeno; BELTRÃO, Renata Paula Lima.

A pele humana tem a capacidade de abrigar microorganismos que podem ser transmitidos pelo contato pele-a-pele, como o que ocorre pelas mãos dos profissionais de saúde, incriminado como uma das principais formas de disseminação das IRAS ${ }^{2}$. A HM seria portanto uma conduta preventiva, rápida, barata e eficaz, onde haveria a remoção das sujidades e dos microrganismos presentes nas mãos ${ }^{4}$.

A Organização Mundial de Saúde (OMS) propõe que a desinfecção das mãos seja realizada em 5 momentos, sendo eles: antes e após o contato com o paciente, antes da realização de procedimentos assépticos, após risco de exposição a fluidos corporais e após o contato com áreas próximas aos pacientes ${ }^{4}$.

Salienta-se que os microrganismos não são transmitidos apenas pelas mãos dos profissionais de saúde, mas também ocorre a transmissão devido a falha na antissepsia de materiais utilizado no tratamento de saúde do paciente e devido ao uso de adornos pelos profissionais ou mesmo pelos visitantes. Algumas medidas são preconizadas para a redução das IRAS, sendo elas: desinfecção das mãos com água e sabão ou solução alcoólica, uso correto dos equipamentos de proteção individual e a limpeza e desinfecção de objetos e ambientes nos serviços de saúde ${ }^{5}$.

Diante disso, o objetivo deste estudo é avaliar como a prática de HM contribui para prevenir a transmissão cruzada de agentes infecciosos na UTI e, por conseguinte, quais as melhores técnicas, produtos e equipamentos para a realização da higienização correta das mãos. Além disso, essa pesquisa tem como intuito avaliar, segundo a literatura, como essa prática é aplicada e monitorada em diversas UTIs.

\section{MATERIAL E MÉTODOS}

O presente estudo trata-se de uma revisão sistemática, elaborada com informações proveniente de artigos obtidos nas bases de dados PUBMED, SCIELO, LILACS e BIREME, mediante utilização de Descritores em Ciência da Saúde- DeCS: "Desinfecção das mãos", "Unidade de Terapia Intensiva" e "Infecção" "Hand disinfection", "Intensive Care Unit" "and" Infection/ "Desinfección de manos", "Unidad de cuidados intensivos" e "Infección) obtidos na Biblioteca virtual em Saúde (BVS), utilizando o operador boleano "AND".

Os critérios de inclusão foram adotados mediante uso de filtros para selecionar: artigos publicados entre 2015 e 2020, estudo em seres humanos e disponibilizados integralmente e de forma gratuita. E os critérios de exclusão foram adotados mediante análise do título e do resumo do artigo, e os estudos que não correspondiam ao tema proposto foram excluídos. 
Na base Scielo, a busca em associação não resultou em artigos, depois de associálos em duplas, ocorreu o seguinte resultado: "Desinfecção das mãos" e "Infecção" foi encontrado 6 artigos; "desinfecção das mãos" e "unidade de terapia intensiva" 3 artigos; "unidade de terapia intensiva" e "infecção" selecionaram 59 artigos. Na base PUBmed, a busca em associação dos 3 descritores não obteve resultados, depois de associá-los em duplas, ocorreu o seguinte resultado: "Desinfecção das mãos" e "Infecção" não obteve resultados; "desinfecção das mãos" e "unidade de terapia intensiva" também não obteve artigos; "unidade de terapia intensiva" e "infecção" 1 artigo. Nas bases LILACS e BIREME a busca em conjunto dos 3 descritores encontrou 1 e 37 artigos, respectivamente. Resultando em 107 artigos.

\section{RESULTADOS E DISCUSSÃO}

Dos 107 artigos, 12 artigos foram excluídos por serem repetidos e 7 porque não foram disponibilizados de forma integral. Em seguida, foi realizada a leitura dos títulos dos 88 artigos restantes, quando 63 foram excluídos porque não se encaixavam no tema. Em seguida, foi feita a leitura dos resumos dos 25 artigos que restaram, sendo excluídos 10 deles, sobrando um total de 15 artigos para a realização da revisão de literatura.

Os pacientes de UTI estão em uso de diversos dispositivos invasivos, o que facilita a disseminação e aquisição de infecções, uma vez que os profissionais de saúde fazem o manuseio desses dispositivos em diversos pacientes durante a rotina laboral. Caso não sejam realizadas técnicas corretas de desinfecção das mãos, podem contribuir para as infecções cruzadas ${ }^{6}$. Segundo o estudo realizado com 51 profissionais que atuavam em UTI, $61 \%$ apresentavam as mãos colonizadas por bactérias patogênicas, sendo identificadas 32 espécies de bactérias diferentes, dentre elas Pseudomonas e acinetobacter, espécies de difícil tratamento ${ }^{2}$.

Dentre os profissionais da equipe de saúde, os técnicos de enfermagem foram os que apresentaram maior nível de microorganismos patogênicos em sua pele, sendo explicado pelo contato mais próximo com os pacientes e suas secreções e pela baixa taxa de adesão desses profissionais à $\mathrm{HM}$, que é cerca de $30 \%$. Em contrapartida, os fisioterapeutas foram os que mais realizavam a HM nos momentos adequados, tendo níveis de adesão acima de 50\%. $2,4,7,8$.

Também foi observado em alguns dos estudos, que muitos profissionais tinham o intuito de promover a HM, mas não dominavam a técnica e acabavam por fazer de forma inadequada e, portanto, menos eficaz, ignoravam a tempo de execução e esqueciam de friccionar determinadas regiões ${ }^{2,4,7,8}$. 
SILVA, Mariane Costa; SERPA, Priscila Gomes Silva; FURTADO, Renata Lima Lages; FURTADO FILHO, Roque Lages; PEREIRA, Ana Karla Galeno; BELTRÃO, Renata Paula Lima.

A adesão das práticas de desinfecção das mãos pelos funcionários de UTI nos 5 momentos preconizados é abaixo de $50 \%{ }^{6,9}$. Um estudo realizado com enfermeiros e técnicos de enfermagem que atuavam em UTI mostrou que dentre os 5 momentos preconizados para a realização de lavagem das mãos, os maiores índices de adesão a essa prática foram nos momentos após o contato direto com o paciente ou com objetos próximos ao leito, levantando a possibilidade de que a equipe priorize a $\mathrm{HM}$ em momentos que julgue com maior potencial de contaminação ${ }^{9}$.

Em contrapartida, o momento de menor adesão é antes do contato com o paciente ou antes da realização de um procedimento. Os profissionais alegam, que devido ao uso da luva, não realizam a desinfecção antes dos procedimentos; todavia, o uso de luvas não substitui a necessidade da $\mathrm{HMI}^{1,7}$. É observado que a taxa de lavagem de mãos tem índices superiores quando o procedimento a ser realizado envolve contato com fluidos corporais, mostrando que existe a preocupação com o autocuidado e o reconhecimento de que as IRAS podem acometer pacientes e profissionais ${ }^{10}$.

Outra fonte de contaminação é a utilização de adornos por profissionais. Em estudo realizado por Ribeiro, foi observado que $28 \%$ dos participantes da pesquisa estavam usando anéis e/ou pulseira e não os removeram antes da realização da HM. O uso desses objetos pode funcionar como reservatórios da microbiota e contribuir para a disseminação das IRAS ${ }^{8}$.

Foi observado que dentre os objetos que estão próximos aos pacientes, os que são mais tocados pelos visitantes e profissionais são: cabeceira do leito, mesa do paciente e os prontuários, podendo propagação de infecções. Deve-se então exercer o controle rigoroso da HM de visitantes e profissionais na entrada e saída da UTI, mesmo quando esses não reconheçam ações e atitudes de potencial desenvolvimento das IRAS ${ }^{11}$.

Os profissionais devem estar em constante aperfeiçoamento, visando a melhoria do cuidado prestado ${ }^{8}$. A falta de adesão à $\mathrm{HM}$ pode estar relacionada a falta de treinamento dos profissionais. ${ }^{2}$ As capacitações servem como forma de relembrar e de atualizar os prestadores de assistência ${ }^{10}$. Foi descrito que após a realização de uma intervenção com os profissionais da UTI sobre a importância da HM foi perceptível a maior adesão ao procedimento ${ }^{12}$. No entanto, o observado é que esses treinamentos não são realizados com satisfatória frequência; em estudo realizado com funcionários de UTI, menos de $50 \%$ dos participantes relataram ter recebido treinamento nos últimos dois anos ${ }^{9}$.

Uma dúvida que muitos profissionais possuem é: qual é o mais indicado, uso de água e sabão ou de preparações alcoólicas? ${ }^{8} \mathrm{O}$ indicado é que seja feito o uso de água e sabão quando as mãos estiverem visivelmente sujas, nos demais usos pode ser feito o uso das 
SILVA, Mariane Costa; SERPA, Priscila Gomes Silva; FURTADO, Renata Lima Lages; FURTADO FILHO, Roque Lages; PEREIRA, Ana Karla Galeno; BELTRÃO, Renata Paula Lima.

1 preparações alcoólicas, no entanto, o uso de álcool não deve ser utilizado por mais de 10 vezes consecutivas, pois pode ocorrer o acúmulo de suor e/ou outras sujidades. Dentre os profissionais dos estudos em análise, foi observado uma preferência pelo uso de água e sabão, sendo este um procedimento que requer um tempo maior, a fricção das mãos com solução alcoólica requer menos tempo e é tão eficaz quanto. Também deve ser levado em conta a qualidade das preparações alcoólicas, estas devem ser entre 60 e $80 \%{ }^{8,10}$.

Uma estratégia é a colocação de dispensários de álcool em gel próximos ao leito de cada paciente, proporcionando aos profissionais facilidade de acesso aos produtos necessários para a higienização das mãos $O$ uso do álcool em gel pode ser mais prática, pois não exige o enxague com água, ou uso de papel toalha para secagem das mãos e leva menos tempo, no entanto, em algumas situações, devem ser tomados alguns cuidados com a qualidade da solução alcoólica, para ser eficaz deve ter concentração entre 60 e 80\% e ainda com a maneira como estas são acondicionadas nas instituições ${ }^{8,13}$.

Algumas instituições podem manter as preparações em embalagens que não protegem o produto, ou usá-las em concentrações fora das faixas indicadas, facilitando a contaminação devido exposição química ou biológica no ambiente ou mesmo pelas mãos dos profissionais que os manipula. Esse estudo realizou cultura de nove dispensários de álcool em gel de uma instituição de saúde, dentre as 140 culturas realizadas, aproximadamente $40 \%$ apresentaram crescimento bacteriano. Essas culturas foram realizadas de 4 partes dos dispensários: tampa, ponto de saída, mangueira e gel, a parte que teve o maior crescimento bacteriano foi a tampa, devido a constante manipulação pelos profissionais na troca do recipiente ${ }^{13}$.

É recomendado que o tempo mínimo para a redução dos microorganismos das mãos utilizando solução alcoólica é que a fricção seja realizada por 20 a 30 segundos. Aproximadamente $60 \%$ dos participantes do estudo, afirmaram friccionar as mãos com álcool em gel por tempo insuficiente para reduzir os microorganismos das mãos ${ }^{14}$.

As principais queixas relatadas pelos profissionais pela falta de hábito de utilizar alcool para a limpeza das mãos foram: que o uso de álcool em gel deixa a mão áspera, ressecada e descamando, alguns relataram ainda parestesia ${ }^{2,15}$. Dentro os motivos dados pela não realização da lavagem das mãos foram citados: esquecimento, poucas pias disponibilizadas nos hospitais, falta de tempo, irritação na pele e falta de materiais ${ }^{8}$.

Pode ser feito o investimento por parte dos hospitais em mais dispensários de álcool, visto que é uma forma mais rápida para a higienização das mãos, esses recipientes devem estar próximo ao leito dos pacientes, no Brasil desde 2010 existe uma lei que torna obrigatório 
SILVA, Mariane Costa; SERPA, Priscila Gomes Silva; FURTADO, Renata Lima Lages; FURTADO FILHO, Roque Lages; PEREIRA, Ana Karla Galeno; BELTRÃO, Renata Paula Lima.

a disponibilidade por parte dos hospitais de dispensários de álcool próximo ao leito dos pacientes. Todos os hospitais possuem pias e dispensários de álcool, principalmente nas áreas onde os pacientes estão instalados. Muitas vezes, perto das pias, existem cartazes ensinando a forma correta de realizar a higienização ${ }^{4,15}$.

\section{CONCLUSÃO}

A HM é uma temática de discussão antiga e que deve ser relembrada corriqueiramente. Práticas inadequadas de lavagem das mãos ou fricção com álcool, tanto por inadequação de técnica ou por frequência, podem implicar em custos e redução da qualidade da assistência, com riscos imensuráveis para os profissionais e para os pacientes. Rever e sedimentar os conceitos básico, pesquisar novas tecnologias e reforçar a relevância da adesão a HM pode manter seguras as UTI do nosso país.

\section{Conflito de interesses}

Não houve conflito de interesses na escrita desse artigo.

\section{REFERÊNCIAS}

1. FERREIRA LL, AZEVEDO LMN, SALVADOR PTCO, MORAIS SHM, PAIVA RM, SANTOS VEP. Cuidado de enfermagem nas Infecções Relacionadas à Assistência à Saúde: Scoping review. Rev Bras Enferm, 2019; 72(2):498-505.

2. SOARES MA, RODRIGUES NM, MENEZES MRO, GERACE DN, DUARTE CR, BRANDÃO PM, BORGES LFA. Microrganismos multirresistentes nas mãos de profissionais de saúde em Unidades de Terapia Intensiva. Rev. Epidemiol. Controle Infecç. Santa Cruz do Sul. 2019; 9(3):187-192.

3. NOGUEIRA LS, FERRETTI-REBUSTINI REL, POVEDA VB, SILVA RCG, BARBOSA RL, OLIVEIRA EM, et al. Carga de trabalho de enfermagem: preditor de infecção relacionada à assistência à saúde na terapia intensiva? Rev Esc Enferm USP. 2015; 49(Esp):36-42

4. SOUZA LM, RAMOS MF, BECKER ESS, MEIRELLES LCS, MONTEIRO SAO. Adesão dos profissionais de terapia intensiva aos cinco momentos da higienização das mãos. Rev Gaúcha Enferm. 2015; 36(4):21-8.

5. PAULA AO, SALGE AKM, PALOS MAP. Infecções relacionadas à assistência em saúde em unidades de terapia intensiva neonatal: uma revisão integrativa. Enfermería Global. 2017; 45. 
SILVA, Mariane Costa; SERPA, Priscila Gomes Silva; FURTADO, Renata Lima Lages; FURTADO FILHO, Roque Lages; PEREIRA, Ana Karla Galeno; BELTRÃO, Renata Paula Lima.

6. ADEGBOYE MB, ZAKARI S, AHMED BA, OLUFEMI GH. Knowledge, awareness and practice of infection control by health care workers in the intensive care units of a tertiary hospital in Nigeria. African Health Sciences. 2018; 18(1).

7. LUCIANO MNF, NASCIMENTO BB, NUNES EM, OLIVEIRA LFM, DAVIM RMB, ALVES ESRC. Adherence to hand hygienize by health professionals in an intensive care unit. J Nurs UFPE on line. 2017; 11(10):3764-70.

8. RIBEIRO FDO, SOUZA MA, PAULA AO, SILVA AG, OLIVEIRA AC. ESTRATÉGIA LÚDICA PARA A MELHORIA DE PRÁTICAS DE HIGIENIZAÇÃO DAS MÃOS ENTRE OS PROFISSIONAIS DE SAÚDE. Rev enferm UFPE on line. 2017; 11(10):3971-9.

9. VASCONCELOS RO, ALVES DCI, FERNANDES LM, OLIVEIRA JLC. Adesão à higienização das mãos pela equipe de enfermagem em unidade de terapia intensiva. Enfermería Global. 2018; 50.

10. OLIVEIRA AC, GAMA, CS, PAULA AO. Adherence and factors related to acceptance of alcohol for antiseptic hand rubbing among nursing professionals. 2017; 51.

11. CHENG VCC, CHAU PH, LEE WM, HO SKY, LEE DWY, WONG SCY, et al. Avaliação por contato com mão-toque de superfícies com toque alto e toque mútuo entre profissionais de saúde, pacientes e visitantes. 2016; 56(1).

12. RODRIGUEZ V, GIUFFRE C, VILLA S, ALMADA G, PRASOPA-PLAIZIER N, GOGNA $M$, GIBBONS L, ELORRIO EG. A multimodal intervention to improve hand hygiene in ICUs in Buenos Aires, Argentina: a stepped wedge trial. International Journal for Quality in Health Care, 2015, 27(5), 405-411

13. KUSAHARA DM, AVELAR AFM, BRAGA AV, MENDES MTM, PETERLINI MAS, PEDREIRA MLG. Contaminação de preparação alcóolica para higienização das mãos em unidade de cuidados intensivos pediátricos. Rev enferm UERJ, Rio de Janeiro, 2016; 24(2):e10640

14. JEZEWSKI GM, LORO MM, HERR GEG, FONTANA RT, AOZANE F, SANTOS FB, KALANKIEWICZ ACB. Conhecimento de profissionais de enfermagem de um hospital privado acerca da higienização das mãos. Rev Cuid 2017; 8(3): 1777-85.

15. OLIVEIRA AO, PAULA AO, GAMA CS. Monitorização da higienização das mãos: observação direta versus taxa autorreportada. Enfermería Global, 2017; 48. 